\title{
A Comparative Study on Linear Effective Properties Predictions of an Argillite Rock Using XFEM
}

\author{
Senjun Wu, Naima Belayachi, Dashnor Hoxha and Duc-Phi Do \\ University of Orléans, INSA-CVL, PRISME, EA 4229, Orléans 45072, France
}

\begin{abstract}
The mechanical behavior of geomaterials is studied using an XFEM (extended finite element method). Usually, the modeling of such heterogeneous material is performed either through an analytical homogenization approach, or numerically, especially for complex microstructures. For comparison, the effective properties are obtained using a classical finite element analysis (through the so-called unit cell method) and an analytical homogenization approach. The use of XFEM proposed here retains the accuracy of the classical finite element approach, allowing one to use meshes that do not necessarily match the physical boundaries of the material constituents. Thanks to such methods, it is then possible to study materials with complex microstructures that have non-simplified assumptions commonly used by other methods, as well as quantify the impact of such simplification. The versatility of XFEM in dealing with complex microstructures, including polycrystalline-like microstructures, is also shown through the role of shape inclusions on the overall effective properties of an argillite rock. Voronoi representation is used to describe the complex microstructure of argillite.
\end{abstract}

Key words: XFEM, micromechanics, argillite, homogenization, Voronoi microstructure, linear behavior.

\section{Introduction}

Heterogeneous geomaterials, like rocks and soils, play a large role in engineering applications. Modeling their behavior accurately is still a challenge. Their macroscopic behavior is complex and strongly influenced by their geological history, their natural composition, and also by complex microstructures resulting in a difference between macroscopic and microscopic scales. Consequently, modeling their behavior accurately is still a challenge.

Basically, nowadays, two alternative and complementary approaches are commonly followed to describe the mechanical behavior of such materials. First, macroscopic models are the models most used in practice and suppose that the material's behavior at any material point is identical to that of a REV (representative elementary volume). In addition, the macroscopic models describe the material behavior

Corresponding author: Naima Belayachi, Ph.D., research fields: non-linear mechanical behavior, micro-macro modeling, coupling modeling and thermo-hydro-mechanical material characterization. E-mail: naima.belayachi@univ-orleans.fr. following a well-established thermodynamic framework [1, 2]. Alternatively, micromechanical models try to establish the constitutive relations in the REV by considering the structure of material and behavior for each of its constituents, a task known as upscaling. In particular, among various analytical micromechanical approaches, the Eshelby inhomogeneous inclusion solution [3] can be used to distinguish between them, including variational approaches allowing for the estimation of the bounds of various parameters [4-6]. In addition, the Mori-Tanaka scheme [7] is another well-known micromechanical approach. Despite the important achievements obtained using such analytical methods in the design of new materials and in the predictions of various effective properties of natural heterogeneous materials, they are limited when it comes to some particular shapes of constituents (most often elliptical or cylindrical) [7].

Thanks to the development of computational techniques, the numerical homogenization method has made considerable progress in taking into account the 
sophisticated behaviors of constituents and complex microstructures of heterogeneous engineering materials. In the majority of published studies, these numerical methods are based on the FEM (finite element method) and make use of the representative unit cell concept as a generalization of periodic materials $[8,9]$.

To simulate a periodic material, some specific boundary conditions, known as periodic boundary conditions, must be applied on the unit cell. These conditions could either be of the constraint displacement type $[8,9]$ or of the free boundary type, and are supposed to reproduce (at least approximately) the conditions of the boundaries of the neighbor cells of a periodic material.

As first proposed by Belytschko and Black [10] and Moës et al. [11], the XFEM (extended finite element method) is established as a serious alternative to the classical FEM for problems of crack propagation without remeshing. Further on, the method was applied to model heterogeneous material, such as a matrix containing inclusions or holes [12]. The description of material discontinuities in the context of XFEM is often realized by the so-called LSM (level-set method) [13]. This numerical technique, initially used for tracking moving interfaces, is able to describe the boundaries of constituents of heterogeneous materials in a simple way, represented by the zero level set curves [12].

In this paper, a homogenization procedure of heterogeneous geomaterials, based on the XFEM approach, similar to that of Moës et al. [11], is used to model the behavior of the argillite of $\mathrm{M} / \mathrm{HM}$ argillite (Meuse Haute Marne), extensively studied in the Underground Research Laboratory of Bure (France) in the framework of geological barrier nuclear waste disposals studies [1,2].

In particular, the effective properties of the argillite rock will be predicted here by considering two microstructure representations: a simplified two-phase matrix-inclusion structure, focusing the analysis on the impact of inclusion and a grain-to-grain microstructure with four phases. This rock, at the mesoscopic level (some millimeters to some centimeters), appears as a composite material with dominating clay-like grains and random mineral (quartz and calcite) grains occupying up to $40 \%$ of the rock's volume, with $2 \%$ of macropores [14].

In contrast to classical FEM numerical analysis with a very limited number of inclusions, in this section, we use a great number of inclusions for some standard spatial distributions with various shapes for which analytical estimators exist [7] in order to verify the well-founded unit cell approach. In addition, the inclusions are used, to some extent, to quantify the errors that could be raised from some practices of numerical and analytical homogenization. We then focused on the evaluation of effective properties and local stress-strain fields of a clay-like rock, using a combination of the XFEM method and a Voronoi tessellation [15-17], which allows for a numerical description of rock microstructure close to that observed in the laboratory [14]. The combination of the capability of XFEM method to modeling complex microstructure and homogenization approach is used in the aim to obtain realistic representation of argillite microstructure, as well as, to study the internal stresses and strains field with more accuracy. The objective is also to study the effect of the inclusions morphology on the effective properties.

In this paper, a bared capital letter (eg., X) refers to a four-order tensor and a bold face symbol (eg., $\mathbf{X}$ ) refers to second-order tensor. A bold face underlined symbol (eg., $\underline{\mathbf{x}}$ ) represents a vector, while an italic symbol (eg., $x$ ) represents a scalar.

\section{Modeling Procedure}

A detailed description of the XFEM is beyond the scope of this paper. However, the interested reader would find a quite complete description of the method in the work of Moës et al. [11], as well as in the references wherein. Nevertheless, for the sake of 
clarity, we briefly present the principle of the XFEM, in addition to the calculation procedures used in this work. The numerical code developed for this purpose, in $2 \mathrm{D}$ and $3 \mathrm{D}$, is a sequence of MATLAB-based routines, following the initial work of Chessa et al. [18].

\subsection{XFEM in Context of an Up-scaling Procedure}

The whole strategy of numerical up-scaling consists of three distinct steps: choosing and meshing an REV (representative elementary volume) of material, describing in some way its structure, solving a simplified problem on this REV, applying suitable loadings and boundary conditions, and calculating averaged values for stress and strains, and deducing values for stiffness coefficients. The principal difference of the XFEM-based homogenization method with a classical approach resides in the first step of the homogenization and concerns both describing the microstructure and constructing an approximated solution.

Generally speaking, the XFEM is a numerical technique that extends the classical FEM approximation by enrichment functions that count for the existence of discontinuities in the structure. Compared to the classical FEM, where the mesh must conform to the surfaces of discontinuity, XFEM uses a uniform mesh to integrate partial differential equations [11]. This mesh is independent of the geometry of the microstructure that facilitates the use of the method in the case of complex geometries and microstructures.

In XFEM, the surfaces of discontinuities (inclusions, cracks and pores...) are taken into account by enriching the finite element approximation with some kind of functions, so that the displacement approximation could be written in the following general form [10, 11]:

$$
\begin{gathered}
\underline{\mathbf{u}}^{\mathrm{XFEM}}=\underline{\mathbf{u}}^{\mathrm{FEM}}+\underline{\mathbf{u}}^{\mathrm{ENR}}= \\
\sum_{i \in I} N_{i}(\underline{\mathbf{x}}) \underline{\mathbf{u}}_{i}+\sum_{J} \underline{\mathbf{a}}_{J} N_{J}(\underline{\mathbf{x}}) F(\underline{\mathbf{x}})
\end{gathered}
$$

where, $I$ is the set of nodes in the mesh, $\underline{\mathbf{u}}_{i}$ is the classical unknown nodal value at the $i$-th node and
$N_{i}(\underline{\mathbf{x}})$ is the classical shape function of FEM approximation; $J$ represents the set of all nodes concerned with enriching approximation, while $\underline{\mathbf{a}}_{J}$ is the set of new degrees of freedom and $F(\underline{\mathbf{x}})$ is the enrichment function. Only the nodes of elements containing a portion of the interface are enriched in order to take into account the discontinuity of displacements through this interface.

When XFEM is used for homogenization purposes, the level set functions are used to describe a boundary between two components and amount to a value associated with that boundary [12]. In particular, they allow for calculating, at each node of the mesh, a value $\phi$ representing the signed distance between the node and the boundary (a negative value within a void or inclusion and a positive value outside).

In the simplest case of a set of $n$ spherical inclusions, for example, the value of this function in a point $\underline{\mathbf{x}}$ is written $[12,19]$ :

$$
\begin{aligned}
& \phi(\underline{\mathbf{x}})=\min \left\{\left\|\underline{\mathbf{x}}-\underline{\mathbf{x}}_{c}^{i}\right\|-r_{c}^{i}\right\} \\
& \underline{\mathbf{x}}_{c}^{i} \in \Omega_{c}^{i} \quad i=1,2, \ldots n
\end{aligned}
$$

where, $\underline{\mathbf{x}}_{c}^{i}$ and $r_{c}^{i}$ are the centre and radius of the $i$-th inclusion, respectively.

It is then possible to calculate an interpolated value of this function at any other point $\underline{\mathbf{x}}$ :

$$
\Phi(\underline{\mathbf{x}})=\sum_{J} N_{J}(\underline{\mathbf{x}}) \phi_{J}
$$

with $N_{J}$ being the shape function associated with Node $J$ of the elements that are cut by interface, and $\phi_{J}$ is the nodal value of the level set function [12].

\subsection{Enrichment Function and Integration of Enriched Elements}

The enrichment function depends on the nature of the problem studied. For example, with a crack analysis problem, the enriched terms $\left(\underline{\mathbf{u}}^{\mathrm{ENR}}\right)$ are chosen in such a way that both jump displacement through the crack and singularity of stress on the crack's tip are considered [10]. More recently, authors used XFEM enrichment displacement to model complex behavior by combining non-linear bridged crack models [20] or 
plasticity dynamic dislocation [21] and advantages of classical XFEM to describe arbitrary cracks.

In the homogenization problem we are dealing with here, the enrichment function for describing the inclusion interface is often constructed using the level set functions. Two enrichment functions for the interface of inclusions have been proposed in previous works $[22,23]$, for a simple case of a $2 \mathrm{D}$ circular inclusion in a reference four nodes quadrilateral element:

$$
\begin{gathered}
F_{1}(\underline{\mathbf{x}})=\left|\sum_{J} \phi_{J} N_{J}(\underline{\mathbf{x}})\right| \\
F_{2}(\underline{\mathbf{x}})=\sum_{J}\left|\phi_{J}\right| N_{J}(\underline{\mathbf{x}})-\left|\sum_{J} \phi_{J} N_{J}(\underline{\mathbf{x}})\right|
\end{gathered}
$$

where, the function $F_{1}(\underline{\mathbf{x}})$ has a 0 value on the interface of inclusions and it reaches its maximum at the boundary of the element, whereas the function $F_{2}(\underline{\mathbf{x}})$ has 0 values at the boundary of the element and in all elements that are not traversed by the interface [23], avoiding the blending errors due to the partially enriched element [18]. In what follows, the function $F_{2}(\underline{\mathbf{x}})$ (Eq. (5)) is used as the enriched function.

Special attention should be paid to the enriched element crossed by the interface where the integration is performed. In fact, on such elements, in as much as Lagrange elements are used for FEM formulation, the gradients of displacement are discontinuous, and therefore, Gauss-Lagrange integration on the whole element is not any more suitable.

In this paper, a separate integration on each side of the interface of an enriched element is performed, as suggested in previous research [22]. For that process, during the integration, the enriched element is divided into sub-elements, each of them fully found in one of the domains separated by the interface. Then, for each convex sub-element, the integration is performed in a classical way, using either four (in 3D case) or three (in 2D case) integration points [22].

\section{Results and Discussions}

In the following sections, we apply this homogenization procedure to study the influence of some microstructure features on the effective elastic properties of a geomaterial. The results obtained by XFEM are compared with classical FEM unit cell-based simulations and/or analytical estimations. For analytical evaluations, we use a Mori-Tanaka scheme [7], while the FEM simulations are performed with ABAQUS.

For simplicity purposes, the problem of influencing the effect of inclusions on effective properties is studied here through the use of $2 \mathrm{D}$ configurations, using a regular mesh of $433 \times 433$ nodes. The analysis will focus on four stiffness coefficients $\left(\mathrm{X}_{1111}, \mathrm{X}_{2222}\right.$, $\mathrm{X}_{1122}$ and $\mathrm{X}_{2211}$ ), obtained by two independent loading tests (respectively in horizontal and vertical directions indicated by indices 1 and 2). More test loadings are necessary to identify all coefficients of stiffness tensor.

\subsection{Effective Properties of Matrix Inclusion Geomaterials with Different Inclusion's Shapes}

In most published results on the role of inclusion's shape on effective properties, particularly for analytical approaches, it is supposed that heterogeneities have sufficient "regular" shapes (more often ellipsoidal or cylindrical). It is supposed that this simplification offers a sufficient accuracy for most practical purposes, even if the question of the effect of this simplification on effective properties is not yet clear.

The impact of inclusion's shape is thought to be even more important in plasticity, where, up until now, numerical methods based on unit cells are considered to be a benchmark to evaluate approximated analytical methods. In the case of unit cells, one chooses a pattern of microstructure that is as close as possible to the reality by using a (very limited) number of inclusions and performing numerical analyses.

There are some published results from some authors where XFEM is already used in the context of the unit cell method [19]. However, using XFEM makes it possible to study this problem with a wide range of inclusion numbers, making it possible to verify the 
well-founded beliefs regarding the unit cell method. In addition, it also makes it possible to eventually quantify, to some extent, the errors introduced by its practice in linear problems. This question is investigated here by XFEM in a number of $2 \mathrm{D}$ plan deformation problems using four different inclusion shapes: circular, elliptical, triangular and non-convex star-like inclusions (Fig. 1).

In order to allow for a comparison of XFEM results and numerical unit cell methods, first, a simplified structure of argillite is considered to be limited in only two phases: the porous clay matrix and mineral inclusions (quartz and calcite are considered identical), represented by calcite inclusions that with properties that are known from published data (Table 1) [9].
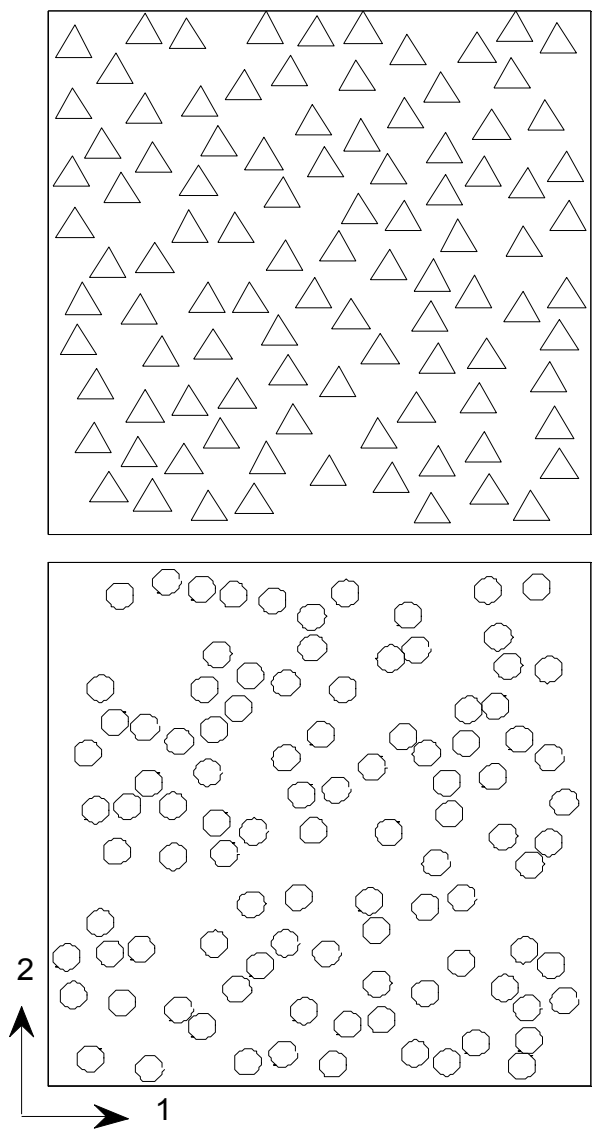

For each shape, a set of random realizations of inclusions has been performed, with each realization containing 100 inclusions. For all cases, the same volume fraction of inclusions is considered $(f=20 \%)$. All circular shape inclusions have the same radius of $25 \mu \mathrm{m}$.

For polygonal and elliptical shapes, the level set function was constructed with the following equation (Eq. 2), which, in this case, is given by:

$$
\begin{aligned}
& \phi(\underline{\mathbf{x}})=\min \left\{\left\|\underline{\mathbf{x}}-\underline{\mathbf{x}}_{c}^{i}\right\|-L\right\} \\
& \mathbf{x}_{c}^{i} \in \Omega_{c}^{i} i=1,2, \ldots n
\end{aligned}
$$

where, $L$ is the distance between the centre $\underline{\mathbf{x}}_{c}^{i}$ and $\underline{\mathbf{x}}_{I}$ ( $\underline{\mathbf{x}}_{I}$ is defined as the intersection between $\left\{\underline{\mathbf{x}}_{c}^{i}-\underline{\mathbf{x}}\right\}$ and the boundary of inclusion), i.e.:
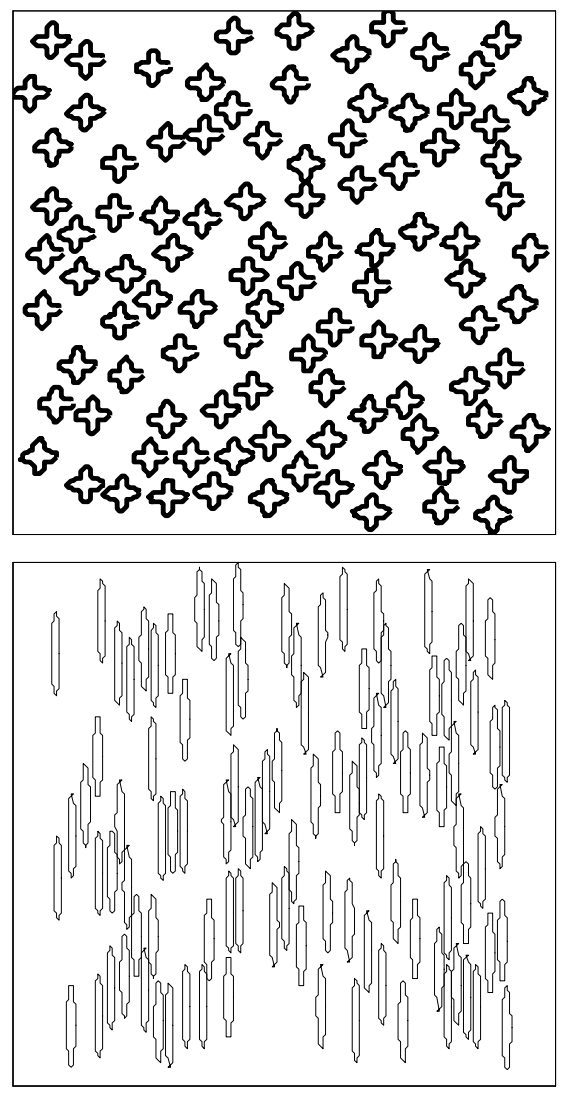

Fig. 1 Shape inclusions used in numerical simulations.

Table 1 Elastic properties of the constituents for two-phase microstructure model.

\begin{tabular}{lll}
\hline Elastic properties & Porous clay matrix & Inclusion \\
\hline Young's modulus & $7,600 \mathrm{MPa}$ & $84,000 \mathrm{MPa}$ \\
Poisson's ratio & 0.1 & 0.3 \\
\hline
\end{tabular}




$$
L=\left\|\underline{\mathbf{x}}_{c}^{i}-\underline{\mathbf{x}}_{I}\right\|
$$

For the elliptical shape inclusion, the semi-axes are $\mathrm{a}_{1}=1 / 3 \times a_{2}, a_{2}=44 \mu \mathrm{m}$ (so that the factor shape is 1/3), respectively. Finally, the triangular-shape inclusions have a side length equal to $68 \mu \mathrm{m}$. For the shapes where the orientation has a sense, a constant orientation is kept during all realizations, so that the randomness concerns only the centers of inclusions.

For the non-convex shapes, the radius $r_{c}^{i}$ in Eq. (2) is replaced by the following function (cylindrical coordinates):

$$
r_{c}(\theta)=r_{0}+a \cos (b \theta)
$$

where, $r_{0}$ is the reference radius, $a$ is the amplitude of oscillation, $b$ indicates the number of oscillations (the following parameters are taken in this work: $r_{0}=24 \mu \mathrm{m}$, $\left.a=0.4 r_{0}, b=4\right)$.

The mean values of the properties in the plane $\left(\mathrm{X}_{1111}\right.$, $\mathrm{X}_{2222}, \mathrm{X}_{1122}$ and $\mathrm{X}_{2211}$ ), obtained from averaging the four realizations of random populations of each shape inclusion, are represented in Table 2.

For these conditions, the differences on effective properties, predicted by the FEM unit cell method and the XFEM predicted values, are small and, in the worst case (for non-convex star-like inclusions), the differences are smaller than $8 \%$. These results seem to justify, to some extent, at least as a first approximation, the use of the unit-cell method in predictions of random materials for which the periodicity is not always verified. Also, it is noted that, in as much as the inclusion's fraction volume is the same, the shape of inclusions, for the cases studied here, has only a moderated impact on the effective mechanical properties. Indeed, even if one considers the $\mathrm{X}_{1111}$ evolution, one could observe that, regardless of the fact that the difference becomes higher with the inclusion shape deviating from the circle, to the non-convex star-like inclusion (with the highest values for this last one shape in Table 2), the differences between circular and non-convex shape inclusions are less than $8 \%$. A significant difference was noted between the XFEM and analytical methods, reaching $15 \%$ for elliptical inclusion because of the elliptical distribution of the Mori-Tanaka scheme.

\subsection{Effective Properties of M/HM Argillite with Grain Voronö Microstructure}

While the matrix-inclusion microstructure model used in the previous section describes quite well the behavior of a great number of geomaterials, in many other cases, a grain-to-grain or a so-called polycrystalline structure is more appropriate to describe

\begin{tabular}{|c|c|c|c|c|c|}
\hline Shape of inclusion & Different methods & $\mathrm{X}_{1111}(\mathrm{MPa})$ & $\mathrm{X}_{2222}(\mathrm{MPa})$ & $\mathrm{X}_{1122}(\mathrm{MPa})$ & $\mathrm{X}_{2211}(\mathrm{MPa})$ \\
\hline \multirow{5}{*}{ Circular inclusion } & XFEM & 10,596 & 10,546 & 1,597 & 1,597 \\
\hline & FEM (ABAQUS) & 10,362 & 10,362 & 1,535 & 1,535 \\
\hline & Relative difference $(\%)$ & 2.2 & 1.7 & 3.9 & 3.8 \\
\hline & Mori-Tanaka & 11,192 & 11,192 & 1,698 & 1,698 \\
\hline & Relative difference $(\%)$ & 5.6 & 6.1 & 6.3 & 6.3 \\
\hline \multirow{5}{*}{ Elliptical inclusion } & XFEM & 9,958 & 12,129 & 1,587 & 1,593 \\
\hline & $\overline{\text { FEM (ABAQUS) }}$ & 9,728 & 11,658 & 1,535 & 1,566 \\
\hline & Relative differences $(\%)$ & 2.3 & 3.8 & 3.2 & 1.6 \\
\hline & Mori-Tanaka & 9,651 & 13,954 & 1,620 & 1,620 \\
\hline & Relative difference $(\%)$ & 3.1 & 15 & 2.1 & 1.7 \\
\hline \multirow{3}{*}{ Triangular inclusion } & XFEM & 10,999 & 10,940 & 1,685 & 1,684 \\
\hline & $\overline{\text { FEM (ABAQUS) }}$ & 10,908 & 10,700 & 1,599 & 1,582 \\
\hline & Relative difference $(\%)$ & 0.8 & 2.2 & 5.1 & 6.0 \\
\hline \multirow{3}{*}{ Non-convex inclusion } & XFEM & 11,068 & 11,077 & 1,717 & 1,715 \\
\hline & $\overline{\text { FEM (ABAQUS) }}$ & 10,701 & 10,701 & 1,589 & 1,589 \\
\hline & Relative difference $(\%)$ & 3.3 & 3.4 & 7.5 & 7.4 \\
\hline
\end{tabular}

Table 2 Effective stiffness coefficients for different shapes of inclusions (matrix-inclusion structure). 
their microstructure. In this section, this grain-to-grain microstructure is obtained using Voronoï tessellations, as is often the case in published works [16, 17].

In order to make a comparison with the results of the matrix inclusion structural model used in the previous section, two-phase Voronoï clay particles and calcite grains are considered using a total of 1,000 grains and a volume fraction of calcite equals to $20 \%$. For each realization, sites were randomly generated, then used as a basis for the Voronoï tessellation. The results obtained using a Voronoï aggregate differ from those obtained using triangular inclusions, with differences that are smaller than 5\% (Table 3). These differences grow to $10 \%$ when comparisons are made between circular inclusions and Voronoï aggregates.

At the level where this study is performed, the rock could be considered a four-phase composite composed by clay particles (enclosing up to $18 \%$ of voids in an inferior scale), quartz and calcite grains and (macro) pores (Table 4). At this level, the volume fractions of these four phases satisfy the unity partition, (i.e., $f_{m}+$ $f_{q u}+f_{c a}+f_{v o}=1$ ), where, the subscripts $m, q u$ and $c a$ stand for clay-like, quartz and calcite grains respectively, and vo stands for macro voids.

While there are some interesting works on poromechanical properties of this rock, all of them are obtained by supposing a simplified structure of the matrix-inclusion type [24]. As previously discussed, this simplification would lead to unjustifiable differences to the reality and a more realistic structure should be considered.

The combination versatility of the Voronoi scheme with the XFEM method seems to be a very good way to obtain stiffness tensor using quite realistic (and representative) polycrystalline-like structures for $\mathrm{M} / \mathrm{HM}$ argillite, as well as to study, in detail, the internal strains and fields due to a dominant compressive stress. In fact, even if this paper is limited to linear mechanical behavior, it is of great interest to examine internal stress and strain fields in order to shed light on the eventual role of rock heterogeneity on the nucleation of sites with high stress and/or strain concentration that could be the origin of the crack initiation and rock damage observed in laboratory.

The volume fractions of constitutive phases of $\mathrm{M} / \mathrm{HM}$ argillite and their mechanical properties are given in Table 4. The behavior of each grain is considered to be a linear elastic one. A compressive mechanical loading of $10 \mathrm{~N}$ is applied on the upper side of the REV (Fig. 2), showing an example of polycrystalline-like microstructure realization, with free lateral faces and vertical constrained displacement on the lower face.

As observed in Table 5, an almost isotropic material is obtained using Voronoi tessellations and random

Table 3 Comparison of effective stiffness coefficients of two-phase Voronoï material with various cases of two-phase matrix-inclusion structures.

\begin{tabular}{lllll}
\hline Shape of inclusion and differences & $\mathrm{X}_{1111}(\mathrm{MPa})$ & $\mathrm{X}_{2222}(\mathrm{MPa})$ & $\mathrm{X}_{1122}(\mathrm{MPa})$ & $\mathrm{X}_{2211}(\mathrm{MPa})$ \\
\hline (1) Circular inclusions & 10,596 & 10,546 & 1,597 & 1,597 \\
(2) Triangular inclusions & 10,999 & 10,940 & 1,685 & 1,684 \\
(3) Two-phase Voronoï & 11,121 & 11,162 & 1,767 & 1,766 \\
Differences between (1) and (3) (\%) & 5.1 & 5.8 & 10.7 & 10.6 \\
Differences between (2) and (3) (\%) & 1.1 & 2.0 & 4.9 & 4.9 \\
\hline
\end{tabular}

Table 4 Elastic properties of the constituents for four-phase microstructure model.

\begin{tabular}{lllll}
\hline Elastic properties & Calcite & Quartz & Porous clay matrix & Macropores \\
\hline Young's modulus (MPa) & 84,000 & 96,400 & 7,600 & 0 \\
Poisson's ratio & 0.3 & 0.08 & 0.1 & 0 \\
Volume fraction & 0.19 & 0.15 & 0.64 & 0.02 \\
\hline
\end{tabular}




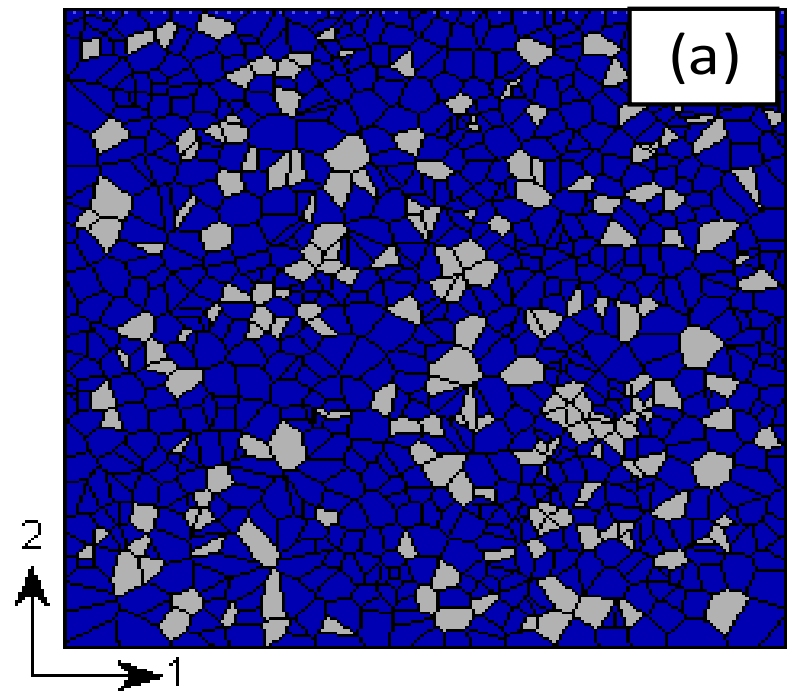

(a)

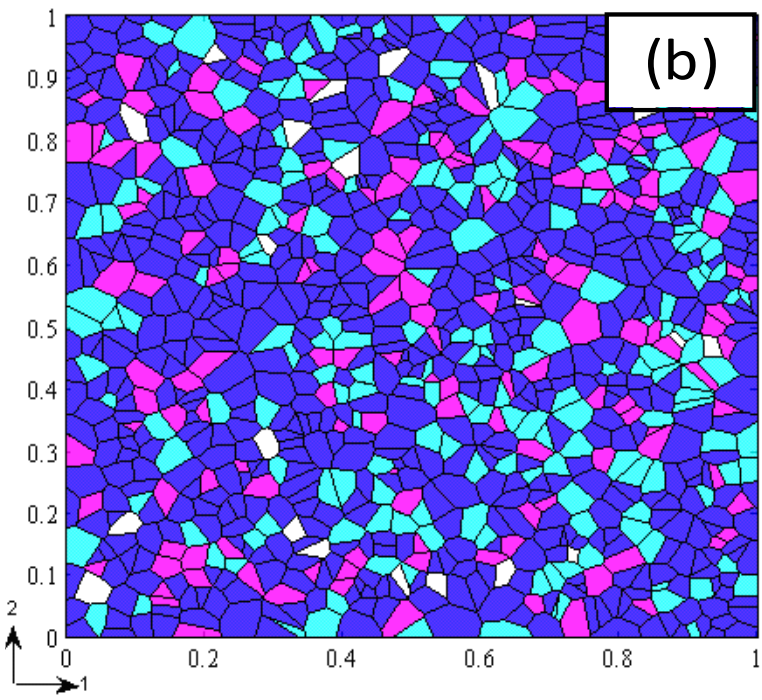

(b)

Fig. 2 A realization of 2D polycrystalline-like microstructure: (a) 2D two-phase Voronoï material (grey sites represent calcite inclusion; blue argillaceous matrix); (b) 2D microstructure of M/HM argillite as a four-phase Voronoï material (blue-clay particles; pink—quartz grains; cyan—calcite grains; white—macropores).

Table 5 Comparison of the effective stiffness coefficients of polycrystalline $\mathrm{M} / \mathrm{HM}$ argillite.

\begin{tabular}{llll}
\hline Effective properties (MPa) & XFEM (four-phase Voronoï) & Mori-Tanaka & Relative differences (\%) \\
\hline $\mathrm{X}_{1111}$ & 14,084 & 12,304 & 12 \\
$\mathrm{X}_{2222}$ & 13,616 & 12,304 & 11 \\
$\mathrm{X}_{1122}$ & 2,632 & 2,284 & 11 \\
$\mathrm{X}_{2211}$ & 2,616 & 2,284 & 11 \\
\hline
\end{tabular}

distributions of grain centers. In this table, the results obtained using polycrystalline-like microstructures of $\mathrm{M} / \mathrm{HM}$ argillite and the XFEM method are presented. In addition, the Mori-Tanaka scheme and a commonly used microstructure of this rock containing an argillaceous matrix and voids and mineral inclusions (calcite and quartz) of cylindrical inclusions are shown.

Beyond the differences due to the not-quite-isotropic behavior of the polycrystalline aggregate, these results show a systematic underestimation of around $11 \%$ of elastic properties from the analytical approach, as compared to direct polycrystalline numerical estimation. This result is important for the assessment of the properties of this rock in situ. In fact, in practice, the properties of clay particles constituting the argillaceous matrix are identified through inverse analytical or semi-analytical analyses, knowing the properties of calcite and quartz, the volume fraction of each phase and the effective properties obtained by classical tests on representative volumes [24]. In light of results obtained using polycrystalline-like structures, it seems that this inverse analytical estimation of the elastic properties of the clay particles would lead to an overestimation of these properties of greater than $10 \%$.

Maps of stress and strain fields in the argillite due to vertical compressive stress are given in Figs. 3 and 4. For the loading conditions and microstructure presented here, the macroscopic stress and strains are $10 \mathrm{MPa}$ and $-0.3 \%$, respectively.

As shown in Figs. 3 and 4, the distribution of stresses and strains is very inhomogeneous and reveals an effect of spatial distribution of the constituents, as well as contacts of grains with the contrasted elastic properties: a high strain gradient is observed around quartz and calcite grains in Fig. 3a. Consequently, these sites of high deformation gradients are the preferential zones of stress concentrations and tensile stress occurrences, as 


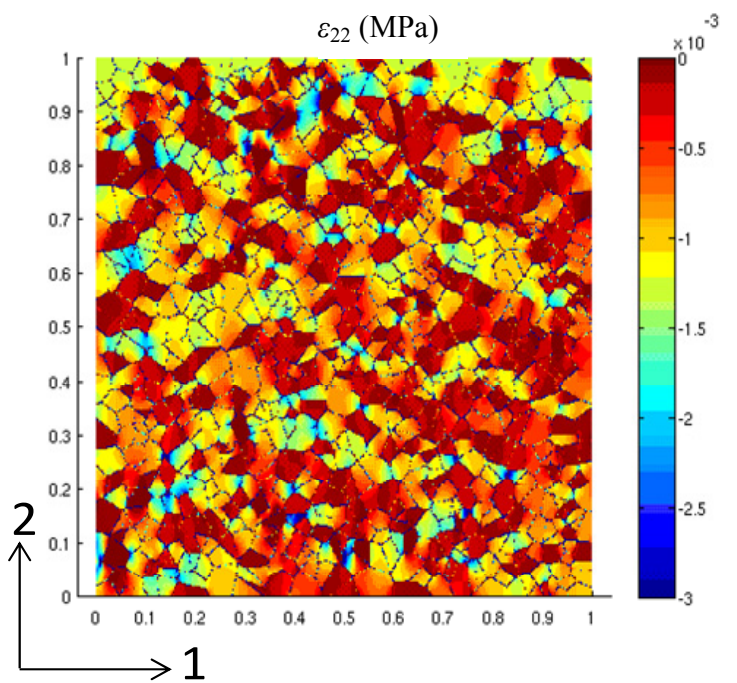

(a)

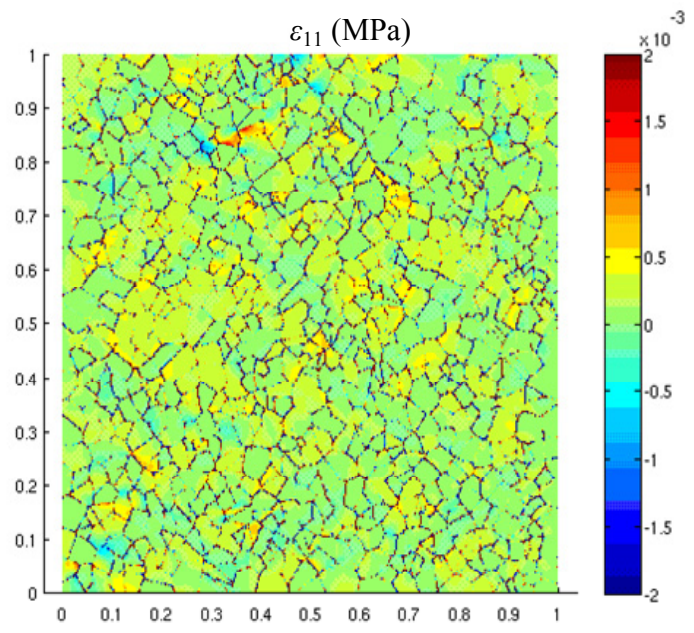

(b)

Fig. 3 Strain fields maps in a 2D polycrystalline microstructure of M/HM due to compressive loading in vertical direction: (a) strain fields in 22 direction ; (b) strain fields in 11 direction.

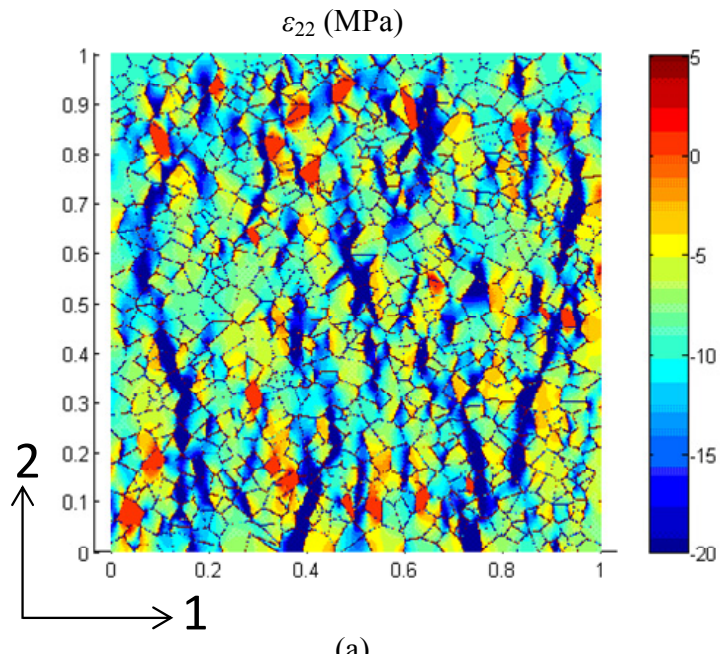

(a)

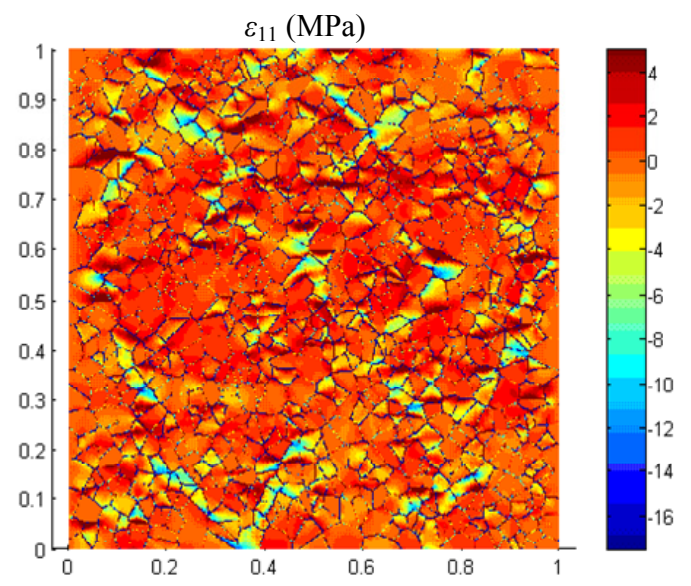

(b)

Fig. 4 Stress field distribution in a $2 \mathrm{D}$ polycrystalline microstructure of $\mathrm{M} / \mathrm{HM}$ due to compressive loading in vertical direction: (a) stress fields in 22 direction; (b) stress fields in 11 direction.

shown in Fig. 4. It is more noteworthy that these tensile stresses appear at the boundaries of the argillaceous grains with those of quartz/calcite (Figs. $4 \mathrm{a}$ and $4 \mathrm{~b}$ ).

While this analysis is quite qualitative, it nevertheless shows that the spatial grain distribution has a significant impact on the local deformation fields and could be the origin of the non-linear elasto-plasto-damage behavior of this rock. A quantitative prediction of damage initiation and evolution under compressive loading needs an understanding of the nonlinear behavior of clay particles and contacts, and constitutes an issue for on-going studies.

\section{Conclusions}

In this paper, we have proposed a numerical homogenization procedure by using the XFEM in the context of the linear mechanical behavior of geomaterials. In addition, the use of level set function ensures to handle efficiently complex geometries with a regular mesh, whereas the use of the finite element method would have induced a new construction of the mesh to each distribution of particles. The various applications have validated the development with the 
XFEM in MATLAB and demonstrated the capability of this method to predict the effective properties of heterogeneous materials such as geomaterials. The XFEM provides similar results to those obtained with classical finite element method and analytical method in many cases, justifying in many situations the use of analytical and periodic unit-cell approaches. However, as it is expected, XFEM simulations demonstrate that neglecting or simplifying the shape of constituents or their spatial distribution, even when a periodic unit cell is used, would lead to differences that could not be any more negligible. In the case of the $\mathrm{M} / \mathrm{HM}$ argillite studied in detail, the differences between XFEM estimations using a polycrystalline-like structure and the Mori-Tanaka, considering the spherical mineral inclusions in an argillaceous matrix, grows $10 \%$ with the Mori-Tanaka systematic underestimation. One could speculate that such differences would be amplified in the case of nonlinear behavior subject of ongoing studies.

By taking into account the structure complexity of a whole REV, the XFEM seems to represent a very serious method for design of composite materials and analyzing of heterogeneous materials in general.

\section{References}

[1] Hoxha, D., Giraud, A., Homand, F., and Auvray, C. 2007. "Saturated and Unsaturated Behaviour Modelling of Meuse-Haute/Marne Argillite." International Journal of Plasticity 23: 733-66.

[2] Chiarelli, S., Shao, J. F., and Hoteit, N. 2003. "Modeling of Elastoplastic Damage Behavior of a Claystone." International Journal of Plasticity 19: 23-45.

[3] Eshelby, J. D. 1957. "The Determination of the Elastic Field of an Ellipsoidal Inclusion, and Related Problems." In Proceedings of the Royal Society of London, 376-96.

[4] Hashin, Z., and Shtrikman, S. 1963. "A Variational Approach to the Theory of the Elastic Behaviour of Multiphase Materials." Journal of the Mechanics and Physics of Solids 11: 127-40.

[5] Castañeda, P. P. 1991. "The Effective Mechanical Properties of Nonlinear Isotropic Composites." Journal of the Mechanics and Physics of Solids 39: 45-71.

[6] Willis, J. R. 1977. "Bounds and Self-consistent Estimates for the Overall Properties of Anisotropic Composites." Journal of the Mechanics and Physics of Solids 25:
185-202.

[7] Mori, T., and Tanaka, K. 1973. "Average Stress in Matrix and Average Elastic Energy of Materials with Misfitting Inclusions." Acta Metallurgica 21: 571-4.

[8] Doghri, I., and Ouaar, A. 2003. "Homogenization of Two-Phase Elasto-Plastic Composite Materials and Structures: Study of Tangent Operators, Cyclic Plasticity and Numerical Algorithms." International Journal of Solids and structures 40: 1681-712.

[9] Belayachi, N., Do, D. P., and Hoxha, D. 2012. "A Note on the Numerical Homogenization of the Mechanical Behavior of an Argillaceous Rock." Computers and Geotechnics 41: 70-8.

[10] Belyschko, T., and Black, T. 1999. "Elastic Crack Growth in Finite Elements with Minimal Remeshing." International Journal for Numerical Methods in Engineering 45: 601-20.

[11] Moës, N., Dolbow, J., and Belytshko, T. 1999. "A Finite Element Method for Crack Growth without Remeshing." International Journal for Numerical Methods in Engineering 46: 131-50.

[12] Sukumar, N., Chopp, D. L., Moës, N., and Belytschko, T. 2001. "Modeling Holes and Inclusions by Levelsets in the Extended Finite-Element Method." Computer Methods in Applied Mechanics and Engineering 190: 6183-200.

[13] Sethian, A. 2000. Level Set Methods and Fast Marching Methods: Evolving Interfaces in Computational Geometry, Fluid Mechanics, Computer Vision, and Materials Science. New York: Cambridge University Press.

[14] Yven, B., Sammartino, S., Geraud, Y., Homand, H., and Villieras, F. 2007. "Mineralogy, Texture and Porosity of Callovo-Oxfordian Argillites of the Meuse/Haute-Marne Region (Eastern Paris Basin)." Mémoires de la Société Géologique de France 178: 73-90.

[15] Ghosh, S., and Moorthy, S. 1995. "Elastic-Plastic Analysis of Arbitrary Heterogeneous Materials with the Voronoï Cell Finite Element Method." Computer Methods in Applied Mechanics and Engineering 121: 373-409.

[16] Forest, S., Barbe, F., and Gailletaud, G. 2000. "Cosserat Modelling of Size Effects in the Mechanical Behaviour of Polycrystals and Multi-phase Materials." International Journal of Solids and Structures 37: 7105-26.

[17] Aurenhammer, F. 1991. "Voronoï Diagrams Survey of a Fundamental Geometric Data Structure." $A C M$ (Association for Computing Machinery) Computing Surveys 23: 345-405.

[18] Chessa, H. W., and Belytschko, T. 2003. "On the Construction of Blending Elements for Local Partition of Unity Enriched Finite Elements." International Journal for Numerical Methods in Engineering 57: 1015-38. 
[19] Yvonnet, J., Quang, H. L., and He, Q. C. 2008. "An XFEM/Level Set Approach to Modeling Surface/Interface Effects and to Computing the Size-Dependent Effective Properties of Nanocomposites." Computational Mechanics 42: 119-31.

[20] Afshar, A., Daneshyar, A., and Mohammadi, S. 2015. "XFEM Analysis of Fiber Bridging in Mixed-Mode Crack Propagation in Composites." Composite Structures 125: 314-27.

[21] Keyhan, M., Goudarzi, M., Mohammadi, S., Roumina, R. 2015. “XFEM-Dislocation Dynamics Multi-scale Modeling of Plasticity and Fracture." Computational Materials Science 104: 98-107.
[22] Dréau, K., Chevaugeon, N., and Moës, N. 2010. "Studied XFEM Enrichment to Handle Material Interfaces with Higher Order Finite Element." Computer Methods in Applied Mechanics and Engineering 199: 1922-36.

[23] Moës, N., Cloirec, M., and Cartraud, P. 2003. "A Computational Approach to Handle Complex Microstructure Geometries." Computer Methods in Applied Mechanics and Engineering 192: 3163-77.

[24] Giraud A, Huynh, Q. V., Hoxha, D., and Kondo, D. 2007. "Application of Results on Eshelby Tensor to the Determination of Effective Poroelastic Properties of Anisotropic Rocks-Like Composites." International Journal of Solids and Structures 44: 3756-72. 